\title{
Experiência da rápida implementação de serviço pioneiro em telessaúde durante a crise da Covid-19
}

\section{Experience of the rapid implementation of a pioneering telehealth service during the COVID-19 crisis}

\author{
Brunnella Alcantara Chagas de Freitas' 1 | $\mid$ brunnella.freitas@ufv.br \\ Wilmara Lopes Fialho' (1) wilmara.fialho@ufv.br \\ Mara Rubia Maciel Cardoso do Prado' (1) $\left.\right|_{\text {mara.prado@ufv.br }}$
}

\begin{abstract}
RESUMO
Introdução: Com base em portaria nacional que regulamenta e operacionaliza a telemedicina como uma das medidas de enfrentamento da emergência de saúde pública decorrente da epidemia de Covid-19, firmou-se uma parceria entre a universidade federal e a gestão municipal para a rápida implementação do serviço de telessaúde para Covid-19.

Relato de Experiência: Trata-se de relato de experiência do processo de implementação do serviço de telessaúde específico para Covid-19, uma parceria entre academia e serviço.

Discussão: O processo de implementação requer dimensionamento da equipe, espaço físico e recursos tecnológicos, treinamento e capacitação contínua da equipe para alinhamento das ações, pactuação e articulação do fluxograma de teleatendimento e telemonitoramento com a rede de saúde local em todos os níveis de atenção, divulgação do serviço para a população, atenção a aspectos éticos e critérios de emissão de documentos, com destaque à apropriação da equipe quanto à abordagem clínica na teleconsulta e no telemonitoramento.
\end{abstract}

Conclusão: A experiência de implementação do serviço Telessaúde Covid em 27 dias relatada neste artigo é passível de replicação por outros municípios como medida de contingenciamento da Covid-19. No relato, destacam-se o número expressivo de atendimentos após dois meses de funcionamento, a missão social da universidade pública e a articulação dela com o sistema de saúde local.

Palavras-chave: Covid-19; Telemedicina; Telessaúde; Saúde Pública; Infecções por Coronavírus.

\begin{abstract}
Introduction: Based on a national ordinance that regulates and operationalizes telemedicine as one of the measures to cope with the public health emergency resulting from the COVID-19 epidemic, a partnership was established between the federal university and municipal management for the rapid implementation of the telehealth service for COVID-19.
\end{abstract}

Experience report: Experience report on the implementation process of the specific telehealth service for COVID-19, a partnership between the academy and the service.

Discussion: The implementation process requires team sizing, physical space and technological resources, training and continuous education of the team to align the actions, for the agreement and articulation of the call center and telemonitoring flowchart with the local health network at all levels of care, disclosure of the service to the population, attention to ethical aspects, criteria for issuing documents, standing out and integration of the team regarding the clinical approach in teleconsultation and telemonitoring.

Conclusion: The experience of implementing the Telehealth Covid service in 27 days is reported in this article, and it is likely to be replicated by other municipalities, as a contingency measure due to COVID-19. The significant number of visits, the social mission of the public university and its articulation with the local health system stand out after two months of operation.

Keywords: COVID-19; Telemedicine; Telehealth; Public Health; Coronavirus infections.

${ }^{1}$ Universidade Federal de Viçosa, Viçosa, Minas Gerais, Brasil.

Editora-chefe: Daniela Chiesa

Editora associada: Rosiane Viana Zuza Diniz

Recebido em 29/06/20; Aceito em 18/01/21.

Avaliado pelo processo de double blind review. 


\section{INTRODUÇÃO}

Diante do cenário de saúde mundial da coronavirus disease 2019 (Covid-19), desde fevereiro de 2020, representantes da Universidade Federal de Viçosa (UFV), da Prefeitura Municipal de Viçosa (PMV), dos serviços de saúde e de diversos setores do município passaram a se reunir para estabelecer estratégias de contingenciamento da doença na região.

Localizado no interior de Minas Gerais, na Região Sudeste do país, mais especificamente na região da Zona da Mata Mineira, o município de Viçosa tem população estimada de 78.846 habitantes (base de 2019) cuja região de saúde compreende dez cidades. A UFV é uma instituição pública com sede localizada em Viçosa.

O Ministério da Saúde, em 20 de março de 2020, publicou a Portaria no 467 que dispõe, em caráter excepcional e temporário, sobre as ações de telemedicina e objetiva regulamentar e operacionalizar as medidas de enfrentamento da emergência de saúde pública de importância internacional decorrente da epidemia de Covid-19. Essa portaria autoriza, em caráter excepcional e temporário, ações de telemedicina, de interação à distância, que podem contemplar atendimento pré-clínico, suporte assistencial, consultas, monitoramento e diagnóstico, realizados por meio de tecnologia da informação e comunicação no âmbito do Sistema Único de Saúde (SUS) ${ }^{1-3}$.

Assim, uma das estratégias, por meio de parceria entre o Departamento de Medicina e Enfermagem da UFV (DEM/UFV) e a Secretaria Municipal de Saúde (SMS), foi a implementação do serviço de telemedicina denominado Telessaúde Covid. O município, em 17 de março de 2020, instituiu um serviço de telessaúde para Covid-19, e, em 27 de março, firmou-se a parceria entre a UFV e PMV para sua ampliação e reestruturação. O processo se concretizou em 22 de abril de 2020, com o nome Telessaúde Covid.

OTelessaúde Covid tem o intuito de prestar atendimento qualificado à distância, por meio de tecnologias, às pessoas com sintomas sugestivos da Covid-19, dúvidas ou história de contato com caso confirmado ou suspeito da doença. No cenário da Covid-19, o serviço exerce papel fundamental ao evitar que o paciente se desloque desnecessariamente, reduzindo a sobrecarga nos serviços de saúde e a propagação do vírus na comunidade, por meio do seu adequado direcionamento, seja para acompanhamento domiciliar ou para avaliação presencial na unidade de saúde apropriada, sempre de acordo com o plano de contingência local ${ }^{1-4}$.

\section{RELATÓRIO DE EXPERIÊNCIA}

\section{$O$ processo para implementação do Telessaúde Covid} Dimensionamento da equipe

Inicialmente, entre os profissionais da rede envolvidos no serviço da SMS, estavam as equipes multiprofissionais do Núcleo de Apoio à Saúde da Família (Nasf) e os integrantes da Equipe Multiprofissional de Atenção Domiciliar (Emad).

O serviço ampliado passou a ser constituído por 16 estagiários bolsistas da SMS (oito estudantes dos dois últimos anos do curso de Medicina e oito do último ano do curso de Enfermagem, todos da UFV), profissionais de saúde do Nasf, professores/preceptores enfermeiros e médicos do DEM, e também médicos e veterinários residentes dos programas de Residência Médica e de Medicina Veterinária da UFV. Em todos os turnos, há sempre a supervisão de um médico e um enfermeiro.

O Telessaúde Covid funciona por 12 horas, todos os dias da semana. As funções da equipe estão distribuídas da seguinte forma: 1 . cinco atendentes que realizam diretamente o teleatendimento e telemonitoramento, sendo quatro estagiários dos cursos de Medicina e Enfermagem e um profissional do Nasf/SMS; 2 . dois supervisores, um médico e um enfermeiro do DEM/UFV, que são professores, técnicos de nível superior ou médicos residentes do Programa de Residência Médica; 3. um regulador, que é médico veterinário do Programa de Residência de Medicina Veterinária da UFV. Existem turnos de seis horas ou 12 horas, a depender do vínculo profissional.

\section{Espaço físico e recursos tecnológicos}

O Telessaúde encontra-se em funcionamento no Laboratório de Informática do DEM/UFV e tem assessoria da diretoria de tecnologia da informação, com possibilidade de expansão. Além da existência de computadores em número suficiente, ampliaram-se os ramais, adquiriram-se headsets e extensões para o supervisor acompanhar a teleconsulta, se necessário, e está programada a aquisição de tablets e WhatsApp para auxiliar a avaliação clínica ${ }^{2,4,5}$.

O Telessaúde Covid realiza as consultas por telefone. A videoconsulta está em implementação e será realizada por WhatsApp quando o atendente e supervisor julgarem necessário, e o paciente tiver recurso tecnológico para tal. O WhatsApp do Telessaúde Covid será exclusivamente para uso em videoconsulta e ficará bloqueado para recebimento de chamadas e mensagens, estando apto apenas para realizar chamadas de vídeo ou enviar e receber documentos quando a equipe julgar necessário (como receita emitida pelo serviço, receber foto de receita do paciente, entre outras situações). Por meio da função WhatsApp Business, podem-se configurar mensagens de ausência e saudação, respostas automáticas e mensagens educativas, e ofertar orientações de forma objetiva, 
além de separar os pacientes por categorias, como é o caso de todos os que serão monitorados de 24 em 24 horas, marcados em vermelho ${ }^{2,4,5}$.

Criou-se um e-mail do serviço para emissão e recebimento de documentos e para comunicação com os demais setores de saúde e com a Vigilância Epidemiológica.

\section{Treinamento e capacitação contínua da equipe para alinha- mento das ações}

O treinamento inicial de toda a equipe foi realizado por videoconferência e complementado pelo apoio presencial da coordenação em alguns turnos no início do serviço. Priorizouse utilizar técnicas de atendimento semelhantes ao presencial, adotando-se o Método Clínico Centrado na Pessoa (MCCP) para a entrevista clínica ${ }^{5,6}$.

Os treinamentos iniciais que visavam às medidas de prevenção da Covid-19 dentro do próprio serviço foram realizados em articulação com os profissionais do DEM/UFV envolvidos no tema.

Mantém-se capacitação contínua da equipe com relação às atualizações emitidas pelas entidades científicas.

Um manual do serviço Telessaúde Covid foi construído pela equipe de coordenação, em consonância com a SMS, e com apoio da Diretoria de Comunicação Institucional da UFV na sua diagramação e divulgação on-line, no site da instituição. O manual visa ao alinhamento das ações do Telessaúde entre a equipe e os diversos setores de saúde, desenhando, dessa forma, o fluxo de atendimento ao paciente dentro da rede de saúde $\mathrm{e}^{2,7-9}$.

\section{Pactuação e articulação do fluxograma de teleatendimento e telemonitoramento com a rede de saúde local em todos os níveis de atenção}

As ações seguem o ciclo PDCA: planejar (plan), fazer (do), verificar (check) e agir (act). As reuniões entre a coordenação do Telessaúde Covid e representantes da gestão de saúde ocorrem semanalmente, bem como o diálogo da coordenação com a equipe do serviço.

Assim, foi pactuado um fluxograma que articula as ações do Telessaúde Covid com os diversos setores de saúde, e também se elaboraram formulários semiestruturados para o teleatendimento e o telemonitoramento. Tudo vem sendo construído e reavaliado continuamente, com base em documentos do Ministério da Saúde e das Secretarias Estaduais de Saúde e nos protocolos de instituições científicas, com adaptação e articulação à rede de saúde local, em todos os níveis de atenção, e de acordo com as pactuações entre a SMS e a UFV 2,4,8,10-15.

\section{Divulgação do Telessaúde Covid para a população}

Vem sendo realizada a ampla divulgação do serviço Telessaúde Covid nos diversos meios de comunicação, com orientações quanto às situações nas quais a população deve telefonar para o serviço. Além disso, há interlocução entre os setores de comunicação da PMV e da UFV para a divulgação de informações de forma coerente.

\section{Aspectos éticos e emissão de documentos}

Em todo atendimento, é perguntado ao paciente se ele autoriza a realização do serviço por telefone. $O$ atendente deve identificar adequadamente o paciente e também se identificar para ele, explicando, no caso de ser estagiário, que está sendo supervisionado por médicos e enfermeiros. Todas as informações obtidas devem ser explicadas ao paciente e compartilhadas com ele, e o atendente deve checar se ficaram bem compreendidas. Os atendimentos devem ser registrados nas folhas de atendimento e monitoramento, que são os prontuários, e, caso a equipe julgue necessário inserir informações adicionais, estas são registradas no modelo de Registro Orientado por Problemas (ReOP), com os dados de identificação do paciente ${ }^{5,16}$.

Se for necessário prescrever medicação, a atendente deverá sempre questionar o paciente sobre alergias medicamentosas, função renal e problemas de saúde prévios, além de orientar sobre possíveis alergias e efeitos colaterais, com registro no prontuário das perguntas e orientações ${ }^{5,16}$.

No caso de envio de documentos necessários ao paciente, eles só poderão ser encaminhados para o WhatsApp ou e-mail da própria pessoa, de outra instituição de saúde (acessado por médico ou enfermeiro) ou outro profissional da saúde (médico ou enfermeiro), visto que são documentos do paciente e o sigilo deve ser mantido. Esses documentos devem, prioritariamente, ter assinatura digital e estar em formato PDF, por questões de segurança ${ }^{17}$.

Caso o paciente seja orientado a ficar em isolamento domiciliar, necessite de atestado para ele ou algum membro do domicílio, ou tenha indicação de Oseltamivir, ele receberá informações sobre a adoção de isolamento adequado e a possibilidade de alergia ou efeitos colaterais da medicação ${ }^{2,9,18,19}$.

O Termo de Responsabilidade de Isolamento Domiciliar e Declaração de Contatos Domiciliares deve ter sua importância e implicações devidamente explicadas pelo atendente, bem como o procedimento para o paciente dar sua anuência. $\mathrm{O}$ paciente deve se orientado sobre o procedimento adotado pela equipe para emissão de documentos (atestados ou receitas), reforçando que estes serão encaminhados após o recebimento do Termo de Responsabilidade de Isolamento Domiciliar e Declaração de Contatos Domiciliares preenchido e consentido por ele via formulário on-line $e^{2,9,18-20}$. 


\section{A abordagem clínica na teleconsulta e no telemonito- ramento}

A teleconsulta possui particularidades quanto à comunicação clínica e ao exame físico, e, por ser realizada por meios tecnológicos de comunicação, deve-se evitar que pareça um "atendimento de telemarketing" e, com isso, um "checklist" impessoal ${ }^{5}$.

É fundamental, inicialmente, estabelecer o motivo do contato telefônico, se teleorientação ou teleconsulta. No caso de tratar-se de uma teleconsulta, devem-se investigar os critérios de gravidade para a adoção precoce de condutas pertinentes. Os seguintes sintomas de bandeira vermelha indicam que $o$ paciente precisa de uma avaliação urgente (pessoalmente ou por vídeo, o que dependerá das circunstâncias clínicas): falta de ar grave ou dificuldade em respirar, dor ou pressão no peito, lábios ou rosto azul e uma história sugestiva de choque (como pele fria e úmida, confusão mental, sonolência ou redução significativa da produção de urina $)^{5}$.

$\mathrm{O}$ atendimento deve seguir uma lógica semelhante à do atendimento presencial, adotando-se, preferencialmente, o MCCP para a entrevista clínica ${ }^{6}$. A seguir, apresentam-se algumas sugestões de comunicação $0^{5,6}$.

O estagiário, profissional ou supervisor, ao atender ao telefone, em revezamento, deve dizer: "Telessaúde Covid, nome do atendente, bom dia/boa tarde/boa noite, em que posso ajudá-lo?". Ato contínuo, verifique com quem está falando, sempre procure falar com o paciente, a não ser que haja algum impedimento para isso (idade, gravidade, dificuldade auditiva); solicite o consentimento dele para que o atendimento/a orientação ocorra pelo telefone; lembre-se de que o formulário é apenas um guia, evite segui-lo como um checklist, e só utilize as perguntas adequadas ao motivo da ligação da pessoa; faça perguntas abertas inicialmente, evitando induziro paciente, como "O que mais?", "Fale-me mais sobre isso,, "O que você quer dizer com falta de ar? Fale-me mais"; use ferramentas como silenciar (com o cuidado para o paciente não achar que a ligação caiu), ecoar e parafrasear; aborde sentimentos, ideias, funcionalidade e expectativas (Sife) quando necessário (por exemplo, paciente insiste em fazer o teste para Covid sem indicação) $)^{5,6}$.

Neste momento pandêmico, as expectativas são frequente motivo de angústia nas pessoas: muitas ligam, por exemplo, apreensivas, com receio de estarem doentes e transmitindo para os entes queridos, outras esperam ser testadas ou que o teleatendimento vá à casa delas fazer um avaliação6.

Faça as perguntas direcionadas que julgar necessárias: "Qual é a sua idade?", "Está com tosse? Dor de garganta? Febre?" (se sim, "Qual temperatura?"), "Como está para respirar? Falta de ar?", "Que dia começou tudo isso?", "Alterações para sentir cheiro e gosto?". Tenha cuidado para não induzir os pacientes nas respostas, nem sempre todas essas perguntas precisam ser feitas, pois o paciente falou, por exemplo, que não tem febre ${ }^{5,6}$.

Caso o paciente desvie o assunto da consulta para algo inapropriado, demonstre que compreende o que ele está expondo e, então, solicite-lhe que retorne à conversa inicial.

Verifique comorbidades prévias, também de maneira aberta, como o exemplo a seguir: "O senhor possui algum problema de saúde?". Se o paciente negar, pergunte: "O senhor faz uso de alguma medicação?". Se o paciente responder que usa um anti-hipertensivo, por exemplo, pode-se perguntar: "O senhor usa essa medicação por qual motivo?". Se ele não souber: "Alguma vez já lhe disseram que o senhor tem pressão alta?"5,6.

Sumarize para o paciente o que você entendeu do que ele the contou e peça-lhe que verifique se não faltou nada. Sempre verifique se o paciente teve contato com caso confirmado ou suspeito de Covid-192,5,6.

Caso o paciente tenha termômetro ou aparelho de aferir pressão arterial, pode-se solicitar que ele confira e informe esses dados no momento da ligação. A avaliação da falta de ar pode ser realizada por perguntas abertas. Eis algumas sugestões: "Como está para respirar?", "Fica cansado para fazer o quê?", "Você está tão cansado que não consegue falar mais do que algumas palavras?", “Você está respirando mais forte ou mais rápido que o normal quando não faz nada?", "Você está tão doente que parou de fazer todas as suas atividades diárias habituais?", "Sua respiração é mais rápida, mais lenta ou igual à normal?", "O que faz você ficar sem fôlego?"15,6.

Se houver algum dado de exame físico que a equipe queira avaliar porvideoconsulta, como a frequência respiratória ou a técnica utilizada pelo paciente para aferir a pressão arterial, isso pode ser realizado pelo tablet com WhatsApp disponível ao lado do computador ${ }^{4,5}$. Nesse caso deve-se, novamente, conferir se o paciente autoriza a videochamada e se domina o uso da ferramenta ou se há alguém em casa que possa ajudá-lo e acompanhá-lo no atendimento - sempre com a anuência do paciente. Reapresente-se para o paciente, confira se ele pode ver e ouvir você adequadamente e conduza o atendimento. Se a conexão estiver ruim, o paciente não dominar a ferramenta, não houver alguém para ajudá-lo e a equipe julgar a necessidade de conferir o exame físico, referencie o paciente para atendimento presencial ${ }^{2,5,6,15}$.

Caso você precise ver algum exame ou receita do paciente, solicite-lhe que envie por e-mail ou WhatsApp lembre-o sempre de identificar-se e comunicar-se de maneira formal, clara e objetiva. Caso haja a indicação de Oseltamivir, verifique a função renal (se há comorbidades que possam comprometê-la), possível alergia medicamentosa e o peso $0^{2,5,18}$.

Caso o paciente seja orientado a ficar em isolamento domiciliar, necessite de atestado para ele ou pessoas do 
domicílio, ou tenha indicação de Oseltamivir, forneça informações sobre o isolamento e a observação de alergia ou efeitos colaterais da medicação. Além disso, oriente-o sobre o procedimento adotado pela equipe para assinatura do documento e recebimento dos arquivos, reforçando que eles serão enviados após a resposta ao formulário 2,4,5.

Abra espaço para as dúvidas do paciente, aborde os sentimentos dele e, se necessário, utilize a ferramenta NURS: chame-o pelo nome (naming), entenda (undestanding), respeite (respecting) e apoie (supporting). Por exemplo, diga: "Percebo que a senhora está chorando" - nesse caso, reserve um tempo para o silêncio ${ }^{21}$.

Esclareça as dúvidas do paciente, se houver. Peça-lhe que repita o que entendeu do que foi conversado. Caso alguma informação tenha ficado desencontrada, esclareça e peça ao paciente que a repita. Despeça-se cordialmente e coloque o serviço à disposição para que, em caso de piora, novos sintomas ou dúvidas, o paciente ligue. Dê as orientações de forma clara e objetiva, e informe sobre o monitoramento que será realizado, se isso for indicado ${ }^{5,6}$.

Ao final, preencha o cabeçalho de identificação. Evite fazer isso no início, para não comprometer a comunicação. Proceda ao registro do atendimento e, se for necessário registrar a evolução, faça isso conforme o ReOP5,16.

Nos casos de telemonitoramento, a mesma lógica deve ser adotada, com adequada identificação da pessoa que realiza a ligação. Informe ao paciente que você está procedendo ao monitoramento e obtenha a conformidade dele. Após a apresentação inicial, pergunte, de maneira aberta, como a pessoa passou desde o último contato com a equipe e lembre-se de que a ficha de atendimento é apenas um guia. Caso o paciente diga que estábem, semnenhum sintoma, evitequestioná-lodetalhadamente sobre cada um dos pontos contidos na ficha. Entretanto, perguntas sobre a manutenção do isolamento ou sintomas em contatos domiciliares devem sempre ser realizadas 2,4,12,20.

\section{Orçamento}

Os custos relativos aos recursos humanos foram assumidos pela SMS (bolsas para os estagiários e profissionais do
Nasf) e pela UFV (médicos, enfermeiros e médicos veterinários). Os custos relativos ao material permanente, equipamentos de proteção individual e de higienização, bem como o espaço físico, foram assumidos pela UFV.

NaTabela 1, estão discriminados os custos com o material permanente para o funcionamento do Telessaúde Covid. Também deve ser contabilizado, para o desenvolvimento das atividades, o custo com equipamentos de proteção individual e de higienização: máscaras cirúrgicas, álcool líquido 70\% para higienização de superfícies e álcool gel 70\% para higienização das mãos.

\section{Experiência inicial}

No contexto atual, o número de casos confirmados/ óbitos no Brasil, em Minas Gerais e em Viçosa é de 1.145.906/52.645, 29.897/720 e 52/0, respectivamente. O primeiro caso confirmado de Covid-19 no município datou de 23 de abril de 2020. Até 23 de junho de 2020, dos 1.651 casos notificados no município, $61,8 \%$ ( $n=1021$ ) foram testados, totalizando 52 casos confirmados. Após dois meses de funcionamento, o Telessaúde realizou 715 novos atendimentos, 417 notificações, perfazendo 25,3\% do total de notificações do município, e um total de 3.632 atendimentos, considerando as ligações recebidas e as efetuadas para telemonitoramento.

$\mathrm{O}$ atendimento segue os princípios do atendimento presencial, adotando-se, preferencialmente, o MCCP para a entrevista clínica. Na experiência do serviço, o tempo médio despendido para o primeiro atendimento é 30 minutos e, para o monitoramento, 15 minutos.

A vigilância ativa e continuada dos pacientes em isolamento domiciliar é fundamental, mantendo-se a comunicação do profissional de saúde com o paciente durante todo o cuidado doméstico até o fim do período de isolamento, com revisão dos sintomas e seguimento evolutivo do quadro. Quando se identifica a necessidade de avaliação presencial do paciente, o Telessaúde Covid agenda a consulta na unidade de saúde de referência, e, quando se constata uma situação grave, providenciam-se a ambulância e a transferência do paciente ao hospital de referência ${ }^{2,4,5,15}$.

Tabela 1. Custos com o material permanente para o funcionamento do Telessaúde

\begin{tabular}{lccc}
\hline \multicolumn{1}{c}{ Item } & Quantidade & Valor unitário (em R\$) & Valor total \\
\hline Computador (desktop avançado) & 10 & $7.282,00$ & $72.820,00$ \\
Tablet, telefone e WhatsApp (aceita chip 3G) & 2 & 560,00 & $1.120,00$ \\
Aparelhos de telefone com fio & 10 & 39,90 & 399,00 \\
Headsets & 10 & 99,00 & 990,00 \\
Total relativo ao material permanente & --- & $7.980,90$ & $75.329,00$ \\
\hline
\end{tabular}

Fonte: Elaborada pelas autoras. 
À luz dos conhecimentos atuais, a telemedicina não deve mais ser considerada uma opção ou um complemento para o sistema de saúde reagir a uma emergência, contudo é necessária a continuidade de estudos para as validações e a superação de barreiras à sua utilização ${ }^{15,22,23}$.

\section{CONCLUSÃO}

A experiência de implementação do serviço Telessaúde Covid em 27 dias relatada neste artigo é passível de replicação por outros municípios como medida de contingenciamento da Covid-19. No relato, destacam-se o número expressivo de atendimentos após dois meses de funcionamento, a missão social da universidade pública e a articulação dela com o sistema de saúde local.

\section{CONTRIBUIÇÃO DAS AUTORAS}

Todos as autoras participaram da coleta e análise dos dados, e da redação e revisão do relato.

\section{CONFLITO DE INTERESSES}

Declaramos não haver conflito de interesses neste estudo.

\section{FINANCIAMENTO}

Declaramos que não houve financiamento neste estudo.

\section{REFERÊNCIAS}

1. Brasil. Portaria no 467, de 20 de março de 2020. Dispõe, em caráter excepcional e temporário, sobre as ações de Telemedicina, com o objetivo de regulamentar e operacionalizar as medidas de enfrentamento da emergência de saúde pública de importância internacional previstas no art. $3^{\circ}$ da Lei no 13.979, de 6 de fevereiro de 2020, decorrente da pandemia de COVID-19 [access in 7 may 2020]. Available from: http://www.planalto.gov. br/CCIVIL_03/Portaria/PRT/Portaria\%20n\%C2\%BA\%20467-20-ms.htm.

2. Brasil. Protocolo de manejo clínico do coronavírus (Covid-19) na atenção primária à saúde. Versão 9. Brasília: Secretaria de Atenção Primária à Saúde; 2020 [access in 7 may 2020]. Available from: https://www.unasus. gov.br/especial/covid19/pdf/37.

3. Bashshur RL, Shannon GW, Krupinski EA, Grigsby J, Kvedar JC, Weinstein RS, et al. National telemedicine initiatives: essential to healthcare reform. Telemed J E Health. 2009;15:600-10.

4. Hollander JE, Carr BG. Virtually perfect? Telemedicine for covid-19. N Engl J Med. 2020;382:1679-81. doi: 10.1056/NEJMp2003539.

5. Greenhalgh T, Koh GCH, Car J. Covid-19: a remote assessment in primary care. BMJ. 2020 Mar 25;368:m1182 [access in 7 may 2020]. Available from: http://www.bmj.com/content/368/bmj.m1182.abstract.

6. Stewart M, Brown JB, McWhinney IR, Weston WW, McWilliam CR, Freeman TR. Medicina Centrada na Pessoa: transformando o método clínico. 3a ed. Porto Alegre: Artmed; 2017.

7. Loeb AE, Rao SS, Ficke JR, Morris CD, Riley 3rd LH, Levin AS. Departmental experience and lessons learned with accelerated introduction of telemedicine during the Covid-19 crisis . J Am Acad Orthop Surg. 2020;28(11):e460-79 [access in 20 jun 2020]. Available from: https:// pubmed.ncbi.nlm.nih.gov/32301818.

8. Universidade Federal do Rio Grande do Sul. Coronavírus (Covid-19): informações para profissionais da APS. Porto Alegre: Faculdade de Medicina; 2020 [access in 7 may 2020]. Available from: https://www.ufrgs. br/telessauders/teleconsultoria/0800-644-6543/\#telecondutas-0800.
9. Freitas BAC, do Prado MRMC, FialhoWL. Telessaúde Covid: teleatendimento e telemonitoramento de pacientes com suspeita ou confirmação de Covid-19. Parceria entre o Departamento de Medicina e Enfermagem da Universidade Federal de Viçosa e a Secretaria Municipal de Saúde de Viçosa-MG. Viçosa; 2020 [access in 2 jun 2020]. Available from: https:// www2.dti.ufv.br/noticias/files/anexos/1591023147.pdf.

10. Minas Gerais. Atualização técnica ao protocolo de infecção humana pelo Sars-Cov-2 nº 02/2020 - 23/03/2020. Definições de casos operacionais e fluxos de testagem laboratorial e notificação dos casos. Belo Horizonte: Secretaria de Estado de Saúde, Centro de Operações de Emergência em Saúde; 2020 [access in 3 apr 2020]. Available from: https://www.saude. mg.gov.br/images/noticias_e_eventos/000_2020/24-03-Atualizacao_ Protocolo-02-Casos-Operacionais-e-Fluxos-de-Testagem-LaboratorialNotificacao-dos-Casos.pdf.

11. Minas Gerais. Atualização técnica ao protocolo de infecção humana pelo Sars-Cov-2 n 05/2020 - 19/05/2020. Definições de casos operacionais e fluxos de testagem laboratorial e notificação dos casos. Belo Horizonte: Secretaria de Estado de Saúde, Centro de Operações de Emergência em Saúde; 2020 [access in 28 jun 2020]. Available from: https://www.itauna. mg.gov.br/arquivos/19-05-atualizacao-protocolo_20115027.pdf.

12. Minas Gerais. Nota técnica Coes Minas Covid-19 no 8 - 20/03/2020 Orientações sobre ações frente ao enfrentamento da infecção humana pelo Sars-Cov-2 (doença pelo coronavírus - Covid-19) para as equipes do Programa Melhor em Casa - Serviço de Atenção Domiciliar. Belo Horizonte: Secretaria de Estado de Saúde, Centro de Operações de Emergência em Saúde; 2020 [access in 20 apr 2020]. Available from: https://www.saude. mg.gov.br/coronavirus/profissionaisdesaude.

13. Espírito Santo. Nota Técnica Covid-19 n 03/2020. Definições para afastamento laboral para profissionais de serviços essenciais. Vitória: Secretaria da Saúde; 2020 [access in 28 jun 2020]. Available from: https:// saude.es.gov.br/Media/sesa/coronavirus/Notas\%20T\%C3\%A9cnicas/ NOTA \%20T\%C3\%89CNICA\%20COVID.19\%20N.\%2003.20.\%20 Defini\%C3\%A7\%C3\%B5es\%20de\%20Afastamento\%20Laboral.pdf.

14. Centers for Disease Control and Prevention. Coronavirus disease 2019 (Covid-19): interim clinical guidance for management of patients with confirmed coronavirus disease (Covid-19). Georgia: Centers for Disease Control and Prevention; 2020 [access in 28 jun 2020]. Available from: https://www.cdc.gov/coronavirus/2019-ncov/hcp/clinical-guidancemanagement-patients.html.

15. Ohannessian R, Duong TA, Odone A. Global telemedicine implementation and integration within health systems to fight the Covid-19 pandemic: a call to action. JMIR Public Health Surveill. 2020;6:e18810 [access in 20 jun 2020]. Disponíve em: https://publichealth.jmir.org/2020/2/e18810.

16. Gusso G, Lopes J.Tratado de medicina de família e comunidade: princípios, formação e prática. 2a ed. Porto Alegre: Artmed; 2018.

17. Conselho Federal de Medicina. Entra em funcionamento serviço que permite validar receitas médicas e atestados digitais. Brasília; 23 abr. 2020 [access in 30 apr 2020]. Available from: http://portal.cfm.org.br/index. php?option=com_content $\&$ view=article \&id=28674:2020-04-23-13-3834\&catid=3\#: :text=O Conselho Federal de Medicina,Telemedicina\%2C emitir atestados ou receitas.

18. Brasil. Parecer Técnico n 67/2020-CGPNI/DEIDT/SVS/MS: indicação, em caráter excepcional, do uso do fosfato de Oseltamivir durante a pandemia da Covid-19. Brasília: Ministério da Saúde, Secretaria de Vigilância em Saúde, Departamento de Imunização e Doenças Transmissíveis, Coordenação-Geral do Programa Nacional de Imunizações; 2020 [access in 18 may 2020]. Available from: https://www.cosemssc.org.br/parecertecnico-no-67-2020-cgpni-deidt-svs-ms/.

19. Grupo Força Colaborativa Covid-19 Brasil. Orientações sobre diagnóstico, tratamento e isolamento de pacientes com Covid-19. 2020 [access in 3 may 2020]. Available from: https://www.infectologia.org.br/ad$\mathrm{min} /$ zcloud/125/2020/04/58d801e961f64463109881311316e4e661d8a1e865fb7638ad61c0827cd83430.pdf.

20. TelessaúdeRS-UFRGS. Orientações para os casos com indicação de isolamento domiciliar. 2020 [access in 15 may 2020]. Available from: https://www.ufrgs.br/telessauders/documentos/telecondutas/material_ isolamento_corona_virus_20200303_ipn_002.pdf. 
21. Cole SA, Bird J. The medical interview: the three function approach. 3rd ed. Philadelphia: Elsevier; 2014.

22. Omboni S. Telemedicine during the Covid-19 in Italy: a missed opportunity? Telemed J E Health. 2020;26(8):973-5. doi: 10.1089/tmj.2020.0106.
23. Dorsey ER, Topol EJ. Telemedicine 2020 and the next decade. Lancet. 2020;395(10227):859 [access in 2 jun 2020]. Available from: https:// www.thelancet.com/journals/lancet/article/PIIS0140-6736(20)30424-4/ fulltext\#.Xtaypz3TtgU.mendeley. 